\title{
Consistency of strain fields and thickness distributions in thermoforming experiments through stereo DIC
}

\section{B. Van Mieghem* - J. Ivens - A. Van Bael}

* Corresponding Author

Bart Van Mieghem

KU Leuven, Department of Materials Engineering, Technology Campus Diepenbeek, Wetenschapspark 27, B3590 Diepenbeek, Belgium

Telephone: $+32(0) 11 / 278825$

Fax: $+32(0) 11 / 278826$

E-mail: bart.vanmieghem@kuleuven.be

http://www.celkunststoffen.be

Jan Ivens

KU Leuven, Department of Materials Engineering, Technology Campus De Nayer Sint-Katelijne-Waver, Jan de Nayerlaan 5, B-2860, Sint-Katelijne-Waver, Belgium

Albert Van Bael

KU Leuven, Department of Materials Engineering, Technology Campus Diepenbeek, Wetenschapspark 27, B3590 Diepenbeek, Belgium

\section{Abstract}

This paper proposes a methodology to determine wall thickness distributions in thermoformed products derived from in-situ surface strain measurements obtained with stereo digital image correlation (DIC), under the assumption of material incompressibility. Wall thickness equations are derived for the Green-Lagrange, Hencky, Biot, logarithmic Euler-Almansi and Euler-Almansi strain definitions and validated with an analytic example. The equations are then used to calculate the wall thickness from digital image correlations with both a commercial software (VIC-3D, version 2012) and an academic software (MatchID3D). Obviously, the choice of the strain definition in the correlation software should not influence the resulting wall thickness values. The comparison reveals that the wall thickness values prove to be identical, regardless of the strain definition that was used, and manual measurements show the validity of the DIC based results. It was also found that, when using VIC-3D version 2009, an earlier release in the software series of Correlated Solutions, all but the GreenLagrange strain definition bring forth unrealistic minimum principal strain values, resulting in different values for the wall thickness.

\section{Keywords}

Digital image correlation, Strain fields, Thermoforming, Thermoplastic, Thickness reduction 


\section{Introduction}

Thermoplastic thermoforming is a generic name for a number of processes that involve the reshaping of a polymer sheet on or in a mould at elevated temperature. Most of the time these processes are vacuum or pressure assisted. Thermoforming is the third most used thermoplastic polymer processing technique next to injection moulding and extrusion. Thermoforming can be used for both unreinforced and (short) fibre reinforced thermoplastics. Reinforced plastics can be shaped using pressure forming or matched die forming, whereas unreinforced plastics can also be shaped by free forming, vacuum forming or drape forming (variant of vacuum forming for male/positive products). Twin sheet forming, a thermoforming variant able to produce hollow products, completes this list as an advanced combination of two vacuum forming / drape forming processes. The thermoforming process variant used in this study is vacuum forming and consists of 4 steps: clamping and heating, blank transfer, forming and simultaneous cooling, and demoulding. Since a cabinet type machine is used, the blank transfer consists of the retraction of the heaters and the upward movement of the mould. In thermoforming, the homogeneity of the wall thickness (distribution) is generally assumed to be the most critical parameter in the quality assessment of a formed product ${ }^{1}$. To assess this quality requirement during the thermoforming process and to be able to optimise the process parameters in an industrial thermoforming environment, in-situ stereo digital image correlation (DIC) is used. This technique has already proven to be valuable to assess the homogeneity of the wall thickness during the different thermoforming process steps ${ }^{2}$.

Digital image correlation is an optical non-contact technique to measure surface displacements and strains that started its development in the 1980 's ${ }^{3-6}$. The term digital image correlation refers to the class of non-contact measurement methods that acquire surface images of an object, store them in digital form, and perform image similarity metrics to extract full-field shape and deformation data. It allows for a sub-pixel measurement accuracy of displacements and strains on complex parts ${ }^{7}$. DIC is widely used in experimental mechanics and in material science. It is one of the most applied optical measurement technologies and demonstrates increasingly broad application prospects ${ }^{8}$. Next to the usage of DIC as such, evaluation of the impact of the used correlation parameters and DIC settings are very diverse ranging from camera calibration ${ }^{9,10}$, speckle patterns and speckling techniques ${ }^{11-13}$, subset sizes and positions ${ }^{14,15}$ to error assessment and uncertainties ${ }^{16-19}$. However, little attention has been given to the differences in resulting thickness obtained with different strain definitions one can use during image correlation.

In this paper, DIC is used to assess the wall thickness distribution of a high impact polystyrene (HIPS: Metzeler Metzoplast SB/HK) vacuum formed partial hemisphere (180 $\mathrm{mm}$ diameter and $65 \mathrm{~mm}$ depth), using different 
strain definitions that are available in two correlation software packages. The results of the academic code MatchID3D V4.1, a correlation platform developed by the research group Mechanics of Materials, Products and Processing of the KU Leuven Technology Campus Ghent, are compared to those obtained by VIC-3D, version 2012, originally developed by researchers at the University of South Carolina. Beginning 2015, the academic MatchID3D code has also been commercialized. The different equations to calculate the thickness, based on the different strain definitions that are available in correlation software in a simple one directional configuration, are developed below. The investigated strain definitions are ${ }^{20-23}$ Green-Lagrange strain (default for large strains), Hencky strain (also called logarithmic, true or natural strain), Biot strain (similar to engineering strain), logarithmic Euler-Almansi strain and Euler-Almansi strain. The latter two are finite strain definitions that are defined in terms of the pure deformation of the translated and rotated material.

The outline of this article is as follows. In section 2 the formalisms that permit to calculate wall thickness based on the different strain definitions is presented. Section 3 describes the experimental procedures. In Section 4, the results of the two digital image correlation software packages are compared and the results obtained with an earlier version of VIC-3D, i.e. version 2009, are elaborated. We conclude in section 5.

\section{Formalism}

In the first part, the thickness equations are derived for the different strain definitions. Next, the equations are validated based on an analytical example i.e. a cross-section of a half-sphere.

\subsection{Strain definitions and wall thickness}

Stereo digital image correlation software typically calculates (3D) displacements. Surface strains are extracted after a local polynomial smoothing (using a so-called strain window) of the displacement data and interpolating the results with a, typically bilinear or quadratic, function. Based on the constant volume assumption these surface strains can be combined to calculate the strain in the thickness direction. A schematic principle of the extraction of the thickness based on stereo DIC applied to thermoforming is shown in Figure $1^{24}$. The volume conservation hypothesis is an approximation since during the thermoforming process the sheet reaches a temperature above the glass transition temperature for amorphous polymers or close to the melting temperature for semi-crystalline polymers and this is associated with a certain thermal expansion. Especially for semicrystalline polymers, the specific volume is therefore affected during heating, forming and cooling. Intermediate 
thickness calculations would therefore be less accurate than thickness calculations after the product reaches its cooled state. Besides, the crystallinity which also influences the thermal expansion is defined by the cooling rate. The validity of the constant volume assumption for the used HIPS grade has been confirmed by comparing initial volume and volume after several annealing cycles of an unclamped HIPS sheet ${ }^{25}$. Moreover, all main dedicated thermoforming simulation software packages rely on the assumption of volume conservation for thickness predictions. Based on this assumption, the different equations to calculate the thickness, based on the different strain definitions that are available in correlation software in a simple cross sectional configuration, are developed below. It is known that the deformation is normally expressed in array form since it is a second order tensor, but for the simplicity and understanding, the following part reduces the problem to a first order problem.

First an imaginary cubical pattern is applied to a flat sheet. The properties of each cube can be defined by its initial length $x$, height $y$ and depth $z$, the latter being the thickness of the sheet. After deformation, one single element can again be defined by its final length $x+d x$, height $y+d y$ and depth $z+d z$ and its deformation and rotation which can be represented in the global coordinate system or in the local coordinate system (Figure 2).

When assuming volume constancy, the following equilibrium applies:

$x y z=(x+d x)(y+d y)(z+d z)$

When calculating strain $(\varepsilon)$ according to the Green-Lagrange strain definition, the general definition is:

$\varepsilon=\frac{1}{2}\left(\lambda^{2}\right.$

where $\lambda$ refers to the ratio of the final length over the initial length; in the $\mathrm{x}$-direction this gives:

$\lambda_{x x}=\frac{x+d x}{x}$

And after substitution:

$\varepsilon_{x x}=\frac{1}{2}\left[\left(\frac{x+d x}{x}\right)^{2} \quad 1\right]$

Similarly, $\varepsilon_{y y}$ and $\varepsilon_{z z}$ can be defined as:

$\varepsilon_{y y}=\frac{1}{2}\left[\begin{array}{ll}\left(\frac{y+d y}{y}\right)^{2} & 1]\end{array}\right.$

$\varepsilon_{z z}=\frac{1}{2}\left[\begin{array}{ll}\left(\frac{z+d z}{z}\right)^{2} & 1]\end{array}\right]$ 
Combining equation 1 with 4, 5 and 6 gives:

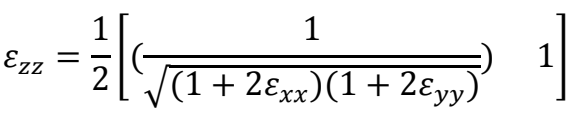

Combining equation 6 with 7, replacing $z+d z$ by $t_{f}, z$ by $t_{i}$ and expressing it in terms of maximum principal strain $\varepsilon_{1}$ and minimum principal strain $\varepsilon_{2}$ gives:

$t_{f}=t_{i}\left(\frac{1}{\sqrt{\left(1+2 \varepsilon_{1}\right)\left(1+2 \varepsilon_{2}\right)}}\right)$

with $t_{f}$, the final wall thickness and $t_{i}$ the initial wall thickness.

A similar approach can be applied to other strain algorithms leading to the following equations for the Hencky (or logarithmic) strain:

$\varepsilon=\ln (\lambda)$

This leads to the following for the final thickness:

$t_{f}=t_{i} e^{\left(-\varepsilon_{1}-\varepsilon_{2}\right)}$

The same equation is valid for the logarithmic Euler-Almansi expression.

The Euler-Almansi strain equation is defined as:

$\varepsilon=\frac{1}{2}\left(1 \quad \frac{1}{\lambda^{2}}\right)$

with corresponding thickness equation:

$t_{f}=t_{i}\left(\sqrt{\left(\begin{array}{ll}1 & 2 \varepsilon_{1}\end{array}\right)\left(\begin{array}{ll}1 & 2 \varepsilon_{2}\end{array}\right)}\right)$

And finally the Biot and Engineering strain definition:

$\varepsilon=\lambda \quad 1$

with corresponding thickness equation:

$t_{f}=t_{i}\left(\frac{1}{\left(1+\varepsilon_{1}\right)\left(1+\varepsilon_{2}\right)}\right)$

The choice of the strain definition is important when comparing strain results to results from strain gauge measurements or simulations but the residual wall thickness should be identical irrespective of the choice of the definition when using the appropriate equation $(8,10,12$ and 14). 


\subsection{Validation of thickness equations}

To demonstrate the effect of the different strain definitions on strain magnitude and on final thickness, the equations are adopted to calculate analytically the strain values and the resulting wall thickness of a cross-section of an imaginary $2 \mathrm{~mm}$ thick sheet that is deformed into a perfect half sphere by means of a hemispherical plug. A cross-section through the centre of the sheet is divided into a finite number of small sections delimited by nodes. It is assumed that all nodes on the initially flat sheet are subject to a vertical projection to the spherical surface of the plug. The boundary nodes are fixed and the nodes closest to the boundary will therefore deform the most. The $\mathrm{z}$-coordinates of the projected nodes $\left(\mathrm{z}_{\mathrm{n}}\right)$ can be calculated by Equation (1):

$z_{n}=\left(\frac{x_{n}}{\tan \left(\sin ^{-1}\left(\frac{x_{n}}{r_{c}}\right)\right)}\right) \quad\left(\begin{array}{ll}r_{c} & c\end{array}\right)$

The radius of the circle is represented by $r_{c}$, the height of the final hemisphere, which not necessarily has to be equal to the radius of the circle, by $h_{c}$. The approximated arc length of a projected segment on the spherical surface is then:

arclengt $\approx \sqrt{\delta z^{2}+\delta x^{2}}$

with $\delta \mathrm{z}=\mathrm{z}_{\mathrm{n}}-\mathrm{z}_{\mathrm{n}+1}$ and $\delta \mathrm{x}=\mathrm{x}_{\mathrm{n}+1}-\mathrm{x}_{\mathrm{n}}$ this leads to following expression for a uniaxial elongation:

$\lambda_{\text {section }}=\frac{\text { arclengt }}{\delta x}$

A visual representation is shown in Figure 3.

Figure 4 shows the radial strain as function of the distance to the centre of the sphere. One can see that the strain at the centre node equals zero and increases with increasing distance from the centre to have a maximum value between the final node and the boundary node. At small strain, all definitions are practically equal, but when increasing, the difference between the different strain definitions gets significantly bigger. The logarithmic increases in an exponential way while the Euler-Almansi strain increases quasi linearly.

The wall thickness based on the different strain definitions is represented in Figure 5. This simple analytical example demonstrates that the residual wall thickness can be calculated by the equations above for either one of the strain algorithms and is consistent irrespective of the choice of the strain definition when assuming volume constancy.

In the following section, it is verified whether the calculated wall thickness values based on DIC measurements are independent of the adopted strain definitions, as they should. 


\section{Experiments}

The first section focuses on the DIC setup on the thermoforming machine. The second section discusses the use of a printed speckle pattern instead of a standard spray-painted pattern.

\subsection{DIC setup}

Two AVT Stingray F-201 cameras with Pentax high resolution, low distortion, $25 \mathrm{~mm}$ lenses are mounted on top of the thermoforming machine at approximately $1190 \mathrm{~mm}$ from the sheet surface. This setup, combined with an aperture f-stop number of $\mathrm{f} / 8$ and two diffuse spotlights mounted in a $45^{\circ}$ angle above the sheet ensures that the sheet will be completely visible in the field of view (FoV: $342 \times 253 \mathrm{~mm}$ ) of the cameras and the necessary depth of field (DoF: $229 \mathrm{~mm}$ ) is achieved ${ }^{26}$. The pixel resolution at this distance is $4.7 \mathrm{px} / \mathrm{mm}$. To be able to judge the effect of the heating and forming stage without having to bother about the influence of the speckle pattern ${ }^{11-15}$, a dotted random, non-repetitive, isotropic and high contrast speckle pattern, necessary for accurate displacement measurements ${ }^{7}$ is printed on $1 \mathrm{~mm}$ thick $330 \times 330 \mathrm{~mm}$ HIPS sheets using a modified inkjet HP Deskjet $1100 \mathrm{C}$ printer. The dots have a diameter of $1 \mathrm{~mm}$, corresponding to a resolution of $4.7 \mathrm{px} / \mathrm{dot}$, as seen by the cameras in undeformed state. To be able to compare the correlation results independently of the calibration results, all images are correlated in both VIC-3D and MatchID3D using identical intrinsic and extrinsic calibration parameters (intrinsic: focal length, principle point, skew, distortion; extrinsic: inter camera position and rotation angles). The correlation settings used in both software packages are tabulated in Table 1. Measures have been taken to achieve high accuracy displacement and strain results ${ }^{8}$. The undeformed blank is a $1 \mathrm{~mm}$ thick HIPS sheet. It is vacuum formed, which is the inverse of the plugging example in previous section, in a partial hemisphere with a radius of $180 \mathrm{~mm}$, a die fillet radius of $5 \mathrm{~mm}$ and a depth of $65 \mathrm{~mm}$ (Figure 6). Digital stereoscopic images are taken at a frame rate of $4 \mathrm{fps}$ during the process.

\subsection{Printed versus sprayed speckle}

For most of the DIC applications, spray painted random speckle patterns are used. Since in this research a printed pattern is used, the correlation accuracy of the printed speckles is compared to that of the spray painted speckles. This is done in a 2D laboratory setup with a single camera, a $25 \mathrm{~mm}$ fixed focal length lens with an aperture f-number of $\mathrm{f} / 1.4$ (small depth of field). The f-number is the ratio of the lens's focal length to the diameter of the entrance pupil. Three sample plates of $100 \times 100 \mathrm{~mm}$ are prepared: one with a lightly sprayed 
pattern (low speckle density), one with a dark sprayed pattern (high speckle density) and one with a dot-printed speckle of $0.78 \mathrm{~mm}$ diameter, which corresponds in this particular setup to a diameter of 29 pixels per dot at a distance from the camera of $150 \mathrm{~mm}$. The dot size is vastly larger than the minimum $3 \times 3$ pixels but artificial compressions up to $130 \%$ are expected in this experiment, so the safety margin is taken proportional to this value. Each of the three plates are positioned successively perpendicular to the lens at a distance of $150 \mathrm{~mm}$. After focusing, a first picture is taken, the plate is moved $1 \mathrm{~mm}$ backwards from the camera and the lens is refocused. This procedure is repeated until the distance between camera and lens reaches $500 \mathrm{~mm}$. The consecutive pictures are correlated with updated reference (incremental) in MatchID2D (the 2D modules of MatchID) using a subset size of 58 pixels (twice the speckle size) and a step size of 9 pixels (1/3th of the speckle size). The calculated average artificial strain (logarithmic Euler-Almansi, strain window 15) values and their corresponding standard deviations over the region of interest, which are in a $2 \mathrm{D}$ setup based solely on the backward movement of the plate and the resulting reduction of the speckle size, are plotted in a chart relative to the distance from the camera (Figure 7). Artificial strain can only be observed in a 2D setup since in a stereo setup the calibration of the cameras and the knowledge of the extrinsic camera parameters would ensure the detection of the imposed backward movement, resulting in rigid body motion instead of increasing strain values. In Figure 7 it can be seen that the results of average artificial strain are identical and independent of the speckling method, while the standard deviation of the printed speckle pattern is even lower than that of the sprayed patterns. This small difference is assumed to be a result from: imperfect focussing after each movement and the uncontrolled speckle (size) distribution in the sprayed samples in combination with the fixed subset size which leads to results that are more likely to deviate from the mean. Artificial strain is used as the correlation quality parameter, since other parameters, such as the average correlation coefficient (R), cannot be used as a qualitative comparison between speckle patterns since it is too much affected by lighting, speckle size, subset size and step size. The correlation coefficient is a measure of the quality of a correlation and the purpose of correlation software is to maximize this coefficient, or to minimize the correlation criterion. Moreover, the correlation coefficient in a correlation using updated references, such as this one, gives an indication of the correlation capabilities between two consecutive pictures but is meaningless when evaluating the overall correlation quality. From these results, it can be concluded that the dot printed pattern, which can be designed according to the needs (size, distribution, density) and making comparison between different experiments insensitive to the speckling, can be used as a good alternative for accurate DIC measurements. 


\section{Results and discussion}

To prove that the approach is not software specific, two correlation software packages are used side by side to assess the wall thickness distribution of a HIPS vacuum formed partial hemisphere. The correlation software packages are VIC-3D and MatchID3D. The first part of the results section deals with VIC-3D version 2012 whereas the second part compares the results of MatchID3D to those of VIC-3D version 2009, which is an earlier release of the same software.

\subsection{Comparison VIC-3D 2012 and MatchID3D V4.1}

Correlation is performed in MatchID3D and VIC-3D, version 2012, using the settings and calibration parameters as described in previous section, and strain and thickness distributions are calculated according to the equations in section 2 .

Since proper strain values can only be calculated when displacements are accurate, the displacement results are validated by loading, meshing, fitting and comparing the resulting point clouds from both software packages in an inspection and mesh processing software, GOM Inspect. Figure 8 shows that both point clouds match well, the local small differences at the border are due to lost correlation (correlation threshold of 0.90) in VIC-3D.

A vertical cross section is considered through the centre point of the final part and the resulting maximum principal $\left(\varepsilon_{l}\right)$ and minimum principal $\left(\varepsilon_{2}\right)$ strains are extracted and displayed as function of the radial position. Maximum principal strains are used instead of strain components $\left(\varepsilon_{x x}\right.$ and $\left.\varepsilon_{y y}\right)$ to eliminate errors caused by missaligning of the coordinate systems between the software packages. By using these strains in the equations as described in section 2, full-field wall thickness distribution is calculated (cf. Figure 1).

Figure 9 and Figure 10 represent the final thickness distribution of the cross-section as calculated by MatchID3D and VIC-3D (2012) respectively, superimposed by a manual thickness measurement on a discrete number of points along the cross section. The manual measurements are performed with a Mitutoyo 209-573 dial calliper gage with a resolution of $0.01 \mathrm{~mm}$ and an accuracy of $0.02 \mathrm{~mm}$ and are the average of 3 repeats. The thickness distributions of both correlation software packages correspond well to the manual measurement and as expected the results of the different strain definitions are identical. This validates the initial assumption and the equations of section 2. It can be noticed that the thickness distribution is not perfectly symmetric. This is caused by a combination of non-uniform heating and material pre-processing history. More details on this phenomenon can be found in some of our previous publications ${ }^{2,25}$ and is hence not discussed in detail. 


\subsection{Comparison VIC-3D 2009 and MatchID3D V4.1}

Since VIC-3D 2012 has been issued only recently and a number of research institutes and companies still use the 2009 version, the exact same experiments were carried out with the older version. When recalculating all different strains in version 2009 based on the correlation results obtained in version 2012, surprisingly completely different results were obtained for the thickness distribution (Figure 11). The thickness calculated on the basis of the Biot strain tensor is omitted since this strain definition was not yet available in version 2009 .

To uncover the reason behind this inconsistency, a closer look is taken at the strain components. Figure 12 to Figure 15 represent the maximum principal and minimum principal strain in the cross section calculated by MatchID3D and VIC-3D version 2009 respectively. Three observations can be made based on these graphs: firstly, the maximum principal strains obtained with both software packages are of the same order of magnitude, but the profiles are not identical (Figure 12 and Figure 13). Secondly, the minimum principal strains differ tremendously, especially when looking at the Euler-Almansi and logarithmic Euler-Almansi strains where very high compressive strains are found for VIC-3D (Figure 15), which is physically meaningless. Thirdly, at the centre point the corresponding strain magnitudes are equal, regardless of the software used. The excessive compression strains for the VIC-3D correlation give unrealistic wall thickness values reaching up to $2.5 \mathrm{~mm}$ for the Euler-Almansi strain and logarithmic Euler-Almansi strain definition, when starting with an initial thickness of $1 \mathrm{~mm}$ (Figure 11). When comparing these results to the thickness profiles calculated with MatchID3D (Figure 9) or VIC-3D version 2012 (Figure 10) and the manual measurements, it can be concluded that the only thickness result in VIC-3D 2009 that corresponds quasi exactly to the reference, is based on the Green-Lagrange strain definition.

The reason for the mismatch in VIC-3D version 2009 is mainly due to exaggerated minimum principal strain components, which the authors believe to be caused by an erroneous conversion of strain data from the local coordinate system to the global coordinate system. This assumption is primarily based on the fact that at the centre point, which is the point that stays quasi orthogonal to the initial position of the sheet and therefore undergoes no rotation, gives realistic results for all strain definitions in VIC-3D version 2009. 


\section{Conclusions}

When using Digital image correlation (DIC) to perform in-situ strain distribution measurements during thermoforming of thermoplastics, and to subsequently determine wall thickness under the assumption of incompressibility, the choice of the strain definition should not influence the resulting wall thickness. In this paper, the use of stereo digital image correlation to define full-field wall thickness distribution of thermoformed products is presented. Wall thickness equations are derived and validated on an analytical example for different strain definitions i.e. the Green-Lagrange, Hencky, Biot, logarithmic Euler-Almansi and Euler-Almansi strain definitions. These equations are then used to calculate the wall thickness from digital image correlations with a commercial software (VIC-3D) and with an academic software (MatchID3D, version 2009 and 2012). The comparison reveals that for MatchID3D and VIC-3D, version 2012, the wall thickness values prove to be identical, regardless of the strain definition that was used. On the other hand in VIC-3D, version 2009, all but the Green-Lagrange strain definition bring forth unrealistic minimum principal strain results, leading to erroneous values for the wall thickness. It is assumed that the errors are caused by an incorrect conversion of strain data from the local coordinate system to the global coordinate system. Apparently Correlated Solutions (VIC-3D) corrected the strain conversion error in the 2012 release of VIC-3D but nevertheless, one should take care when using or interpreting experimental strain data generated in VIC-3D version 2009 when it involves all other strain algorithms than Green-Lagrange. The authors would like to stress on the fact that all publications involving digital image correlation should include, next to the correlation and setup settings necessary for the evaluation of the measurement accuracy, the brand name and version of the correlation software.

\section{Acknowledgements}

The authors would like to thank Pascal Lava from the MeM2P group of KU Leuven Technology Campus Ghent for his technical support with MatchID3D. 


\section{References}

1. Throne JL. Technology of thermoforming: Hanser; 1996.

2. Van Mieghem B, Lava P, Debruyne D, Van Bael A, Ivens J. Digital image correlation for online wall thickness measurements in thick gauge thermoforming. Key Engineering Materials. 554557:1583-1591, 2013.

3. Peters WH, Ranson WF. Digital imaging techniques in experimental stress-analysis. Optical Engineering. 21(3):427-431; 1982.

4. Peters WH, Ranson WF, Sutton MA, Chu TC, Anderson J. Application of digital correlation methods to rigid body mechanics. Optical Engineering. 22(6):738-742; 1983.

5. Chu TC, Ranson WF, Sutton MA, Peters WH. Applications of digital-image-correlation techniques to experimental mechanics. Experimental Mechanics. 25(3):232-244; 1985.

6. Sutton MA, Cheng MQ, Peters WH, Chao YJ, McNeill SR. Application of an optimized digital correlation method to planar deformation analysis. Image and Vision Computing. 4(3):143-150; 1986.

7. Sutton MA, Orteu J, Schreier H. Image correlation for shape, motion and deformation measurements: Springer; 2009.

8. Pan B, Qian KM, Xie HM, Asundi A. Two-dimensional digital image correlation for in-plane displacement and strain measurement: a review. Measurement Science and Technology. 20(6); 2009.

9. Zhang ZY. A flexible new technique for camera calibration. Ieee Transactions on Pattern Analysis and Machine Intelligence. 22(11):1330-1334; 2000.

10. Patterson EA, Hack E, Brailly P, et al. Calibration and evaluation of optical systems for fullfield strain measurement. Optics and Lasers in Engineering. 45(5):550-564; 2007.

11. Pan B, Lu ZX, Xie HM. Mean intensity gradient: An effective global parameter for quality assessment of the speckle patterns used in digital image correlation. Optics and Lasers in Engineering. 48(4):469-477; 2010.

12. Lecompte D, Smits A, Bossuyt S, et al. Quality assessment of speckle patterns for digital image correlation. Optics and Lasers in Engineering. 44(11):1132-1145; 2006.

13. Hua T, Xie HM, Wang SM, Hu ZX, Chen PW, Zhang QM. Evaluation of the quality of a speckle pattern in the digital image correlation method by mean subset fluctuation. Optics and Laser Technology. 43(1):9-13; 2011.

14. Yaofeng S, Pang JHL. Study of optimal subset size in digital image correlation of speckle pattern images. Optics and Lasers in Engineering. 45(9):967-974; 2007.

15. Pan B, Xie H, Wang Z, Qian K, Wang Z. Study on subset size selection in digital image correlation for speckle patterns. Optics Express. 16(10):7037-7048; 2008.

16. Lava P, Cooreman S, Coppieters S, De Strycker M, Debruyne D. Assessment of measuring errors in DIC using deformation fields generated by plastic FEA. Optics and Lasers in Engineering. 47(7-8):747-753; 2009.

17. Lava P, Cooreman S, Debruyne D. Study of systematic errors in strain fields obtained via DIC using heterogeneous deformation generated by plastic FEA. Optics and Lasers in Engineering. 48(4):457-468; 2010.

18. Lava P, Coppieters S, Wang Y, Van Houtte P, Debruyne D. Error estimation in measuring strain fields with DIC on planar sheet metal specimens with a non-perpendicular camera alignment. Optics and Lasers in Engineering. 49(1):57-65; 2011.

19. Wang YQ, Sutton MA, Bruck HA, Schreier HW. Quantitative error assessment in pattern matching: effects of intensity pattern noise, interpolation, strain and image contrast on motion measurements. Strain. 45(2):160-178; 2009.

20. Lava P, Cooreman S, Coppieters S, Debruyne D. Sources of systematic errors in the determination of heterogeneous strain fields obtained via DIC. Proceedings of SEM Annual Conference; 271-281; 2010.

21. Biot MA. Mechanics of incremental deformations. London: J. Wiley \& Sons; 1964.

22. Malvern LE. Introduction to the mechanics of a continuous medium. Englewood Cliffs, New Jersey: Prentice-Hall Inc.; 1969. 
23. Kaye A, Stepto RFT, Work WJ, Aleman JV, Malkin AY. Definition of terms relating to the non-ultimate mechanical properties of polymers. Pure and Applied Chemistry. 70(3):701-754; 1998.

24. Van Mieghem B, Hamblok M, Van Bael A, et al. On the potential of stereo digital image correlation in thermoforming. Thermoforming Quarterly. 34(2):20-28; 2015.

25. Van Mieghem B, Van Bael A, Ivens J. Impact assessment of extrusion anisotropy on quality of thermoformed products. Proceedings of Composites Week@ @euven and TEXCOMP-11 Conference. Leuven, Belgium; 1-8; 2013.

26. Greenleaf AR. Photographic optics. New York: The Macmillan Company; 1950. 


\section{Figures}
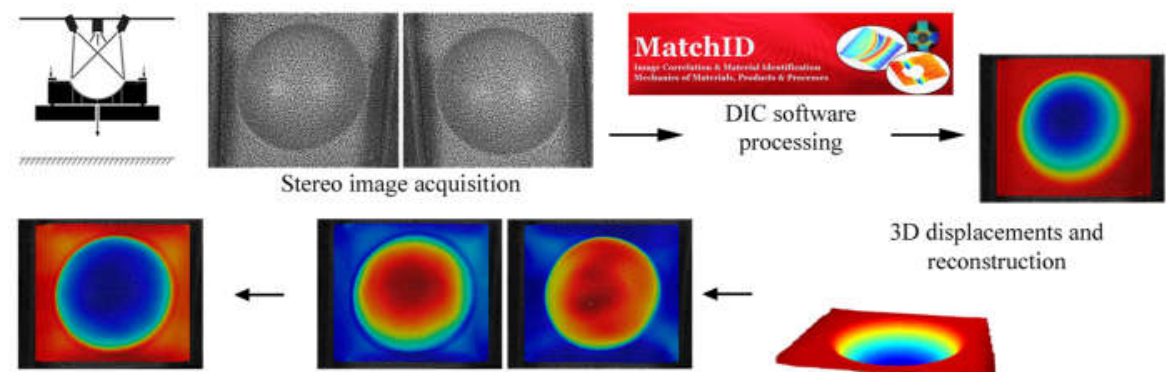

3D displacements and reconstruction

Wall thickness

Min. and max. principal strain

Figure 1. Principle of thickness definition based on stereo DIC measurements

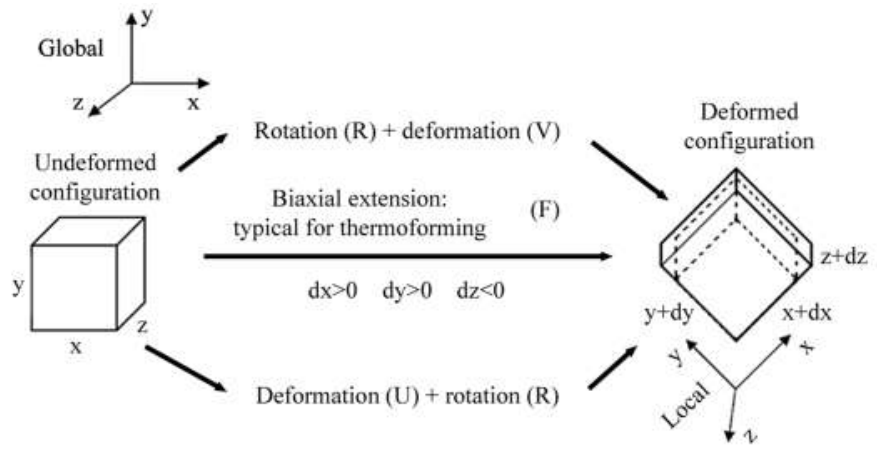

Figure 2. Schematic representation of undeformed and deformed conventions

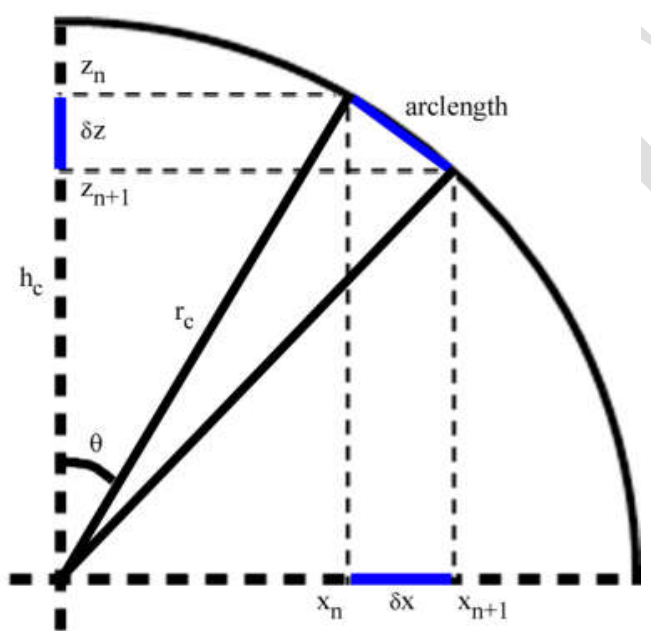

Figure 3. Elongation based on vertical projection on a spherical surface 


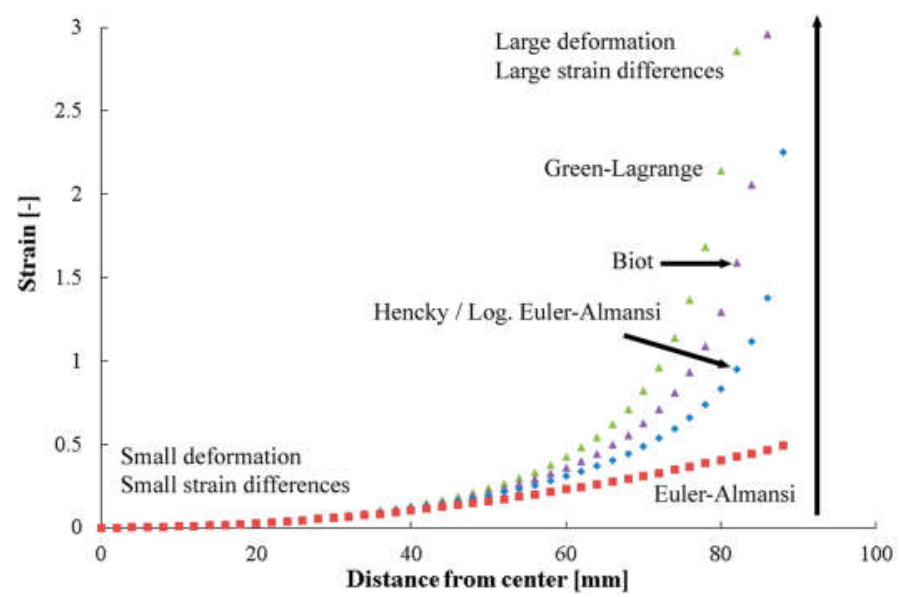

Figure 4. Strain magnitudes for the different strain algorithms in a cross-section of a half-sphere with a radius of $90 \mathrm{~mm}$ as function of the distance to the centre

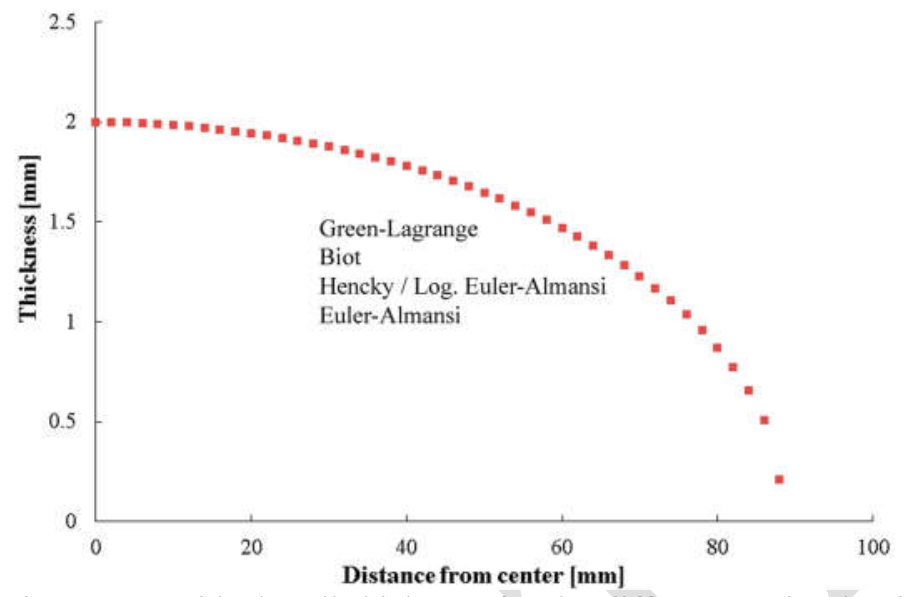

Figure 5. Residual wall thickness for the different strain algorithms in a cross-section of a half-sphere with a radius of $90 \mathrm{~mm}$ as function of the distance to the centre
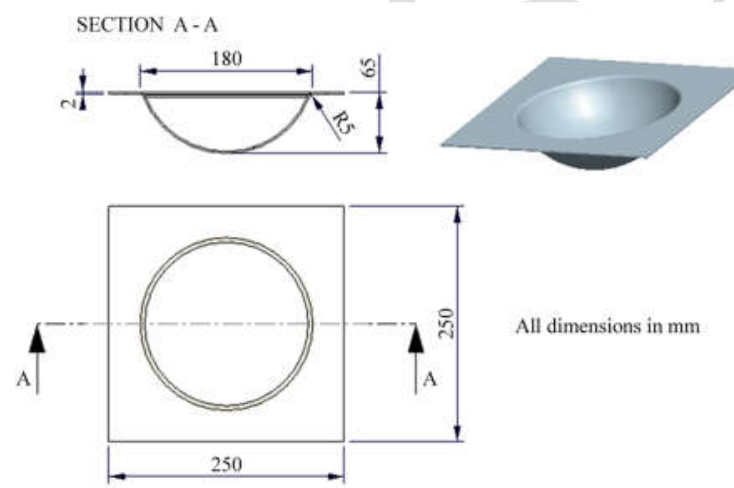

Figure 6. Partial hemispheric mould used for the vacuum forming 


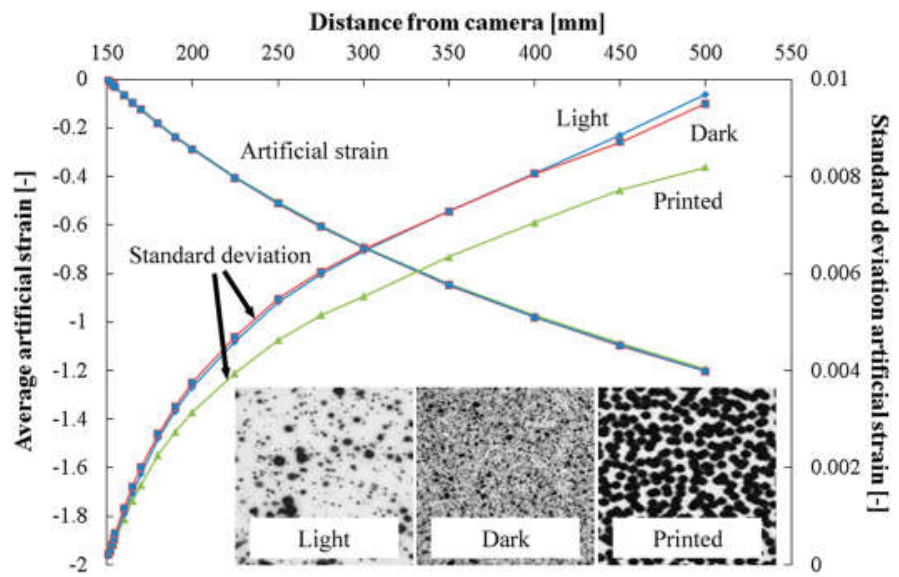

Figure 7. Average artificial strain and standard deviation as function of the camera distance for three different speckle patterns (decreasing curves: artificial strain, increasing curves: standard deviation of artificial strain)
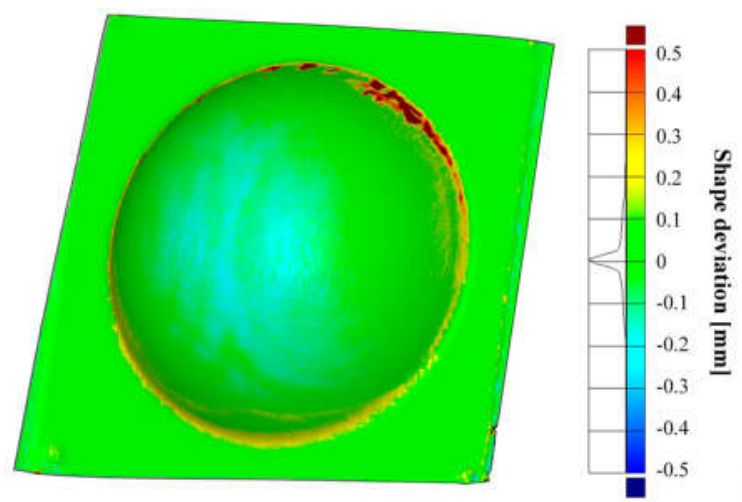

Figure 8. Mesh matching between VIC-3D and MatchID3D point clouds show good agreement

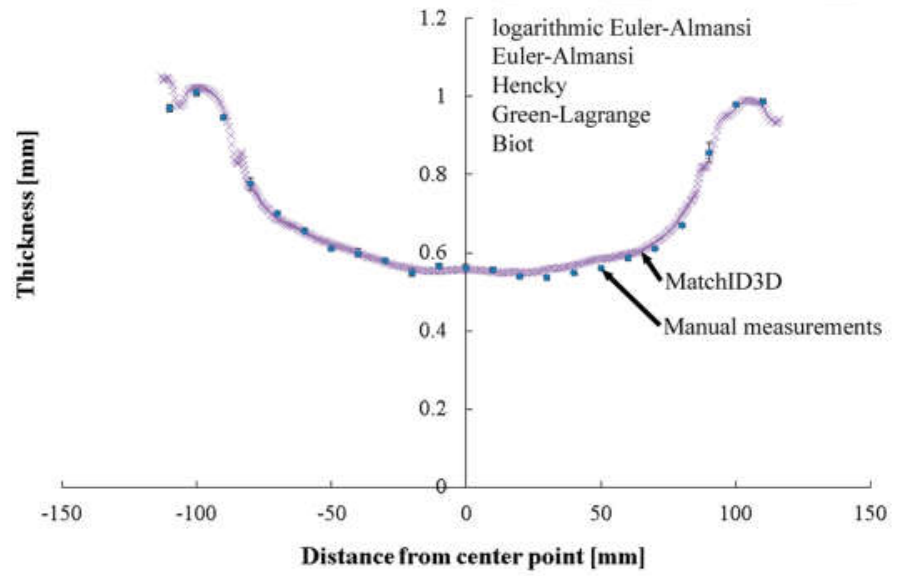

Figure 9. Thickness of a cross section as calculated by MatchID3D superimposed with the manually measured thickness profile 


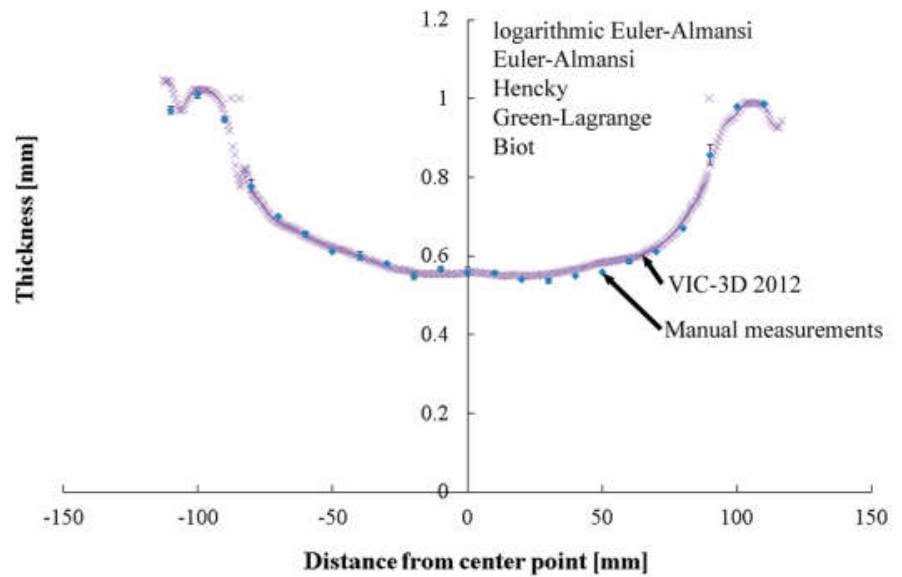

Figure 10. Thickness of a cross section as calculated by VIC-3D 2012 superimposed with the manually measured thickness profile

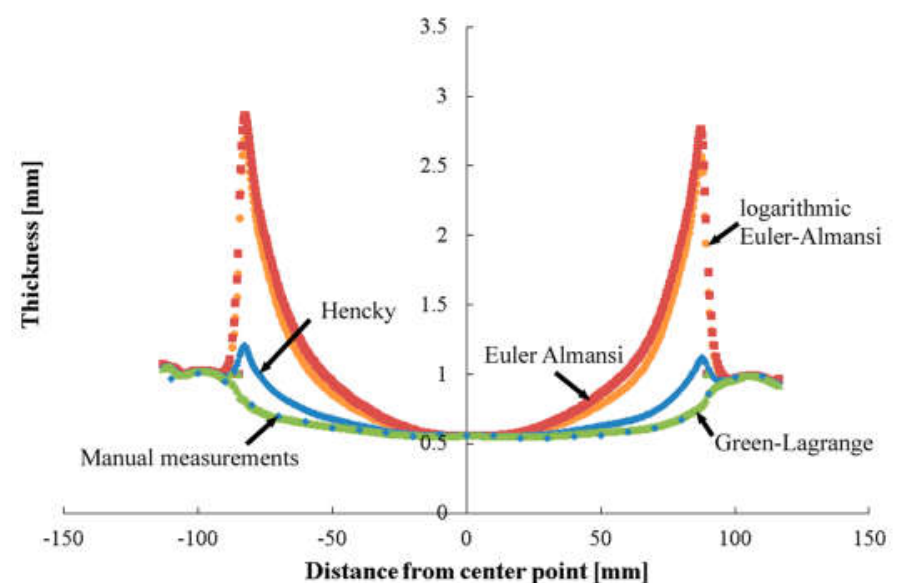

Figure 11. Thickness of a cross section as calculated by VIC-3D 2009 superimposed with the manually measured thickness profile

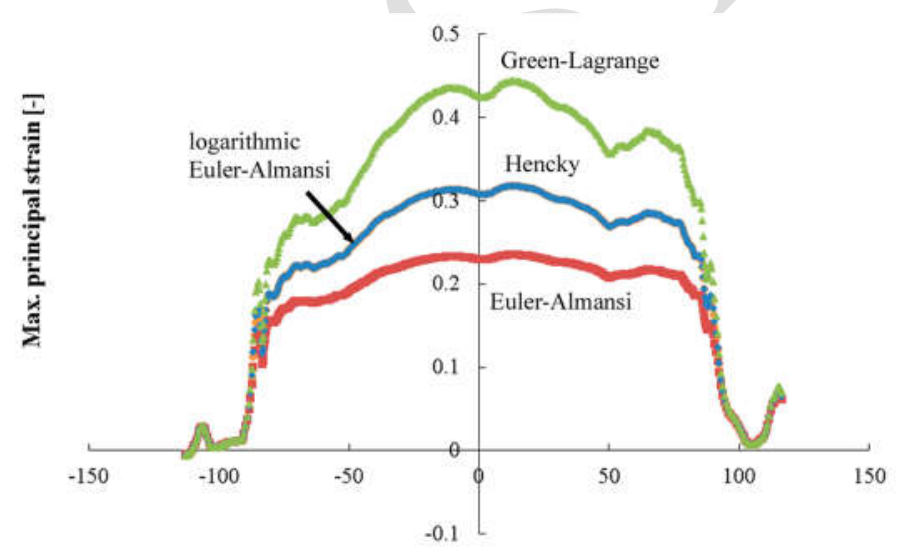

Distance from center point [mm]

Figure 12. Maximum principal strain as calculated by MatchID3D as function of the distance to the centre 


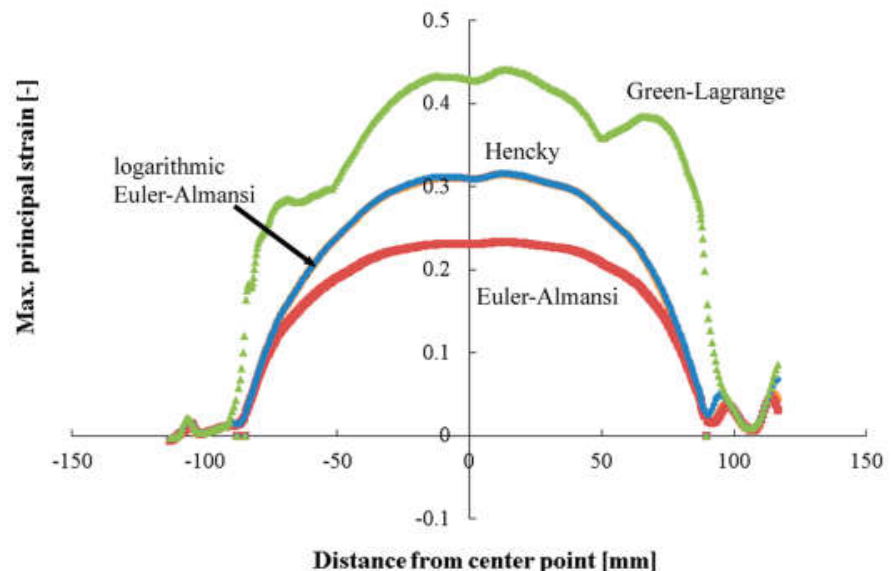

Figure 13. Maximum principal strain as calculated by VIC-3D 2009 as function of the distance to the centre

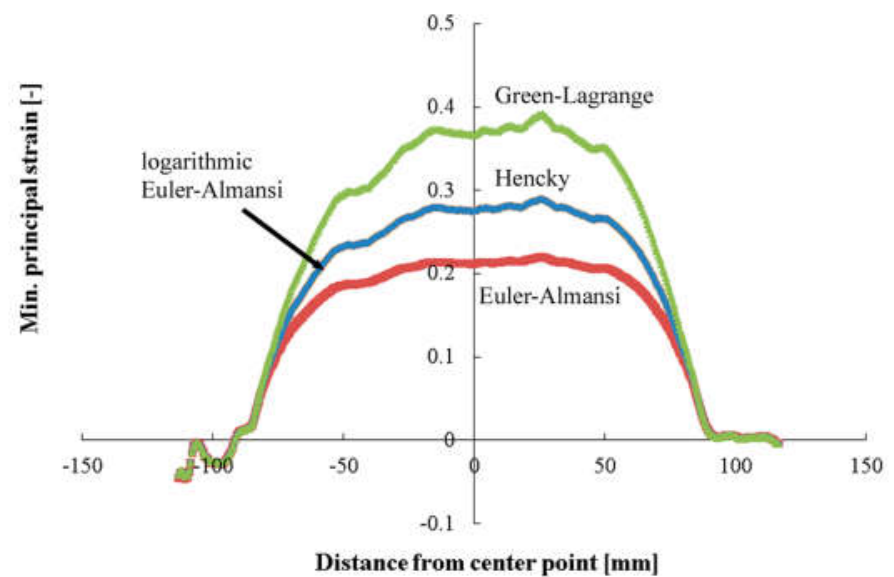

Figure 14. Minimum principal strain as calculated by MatchID3D as function of the distance to the centre

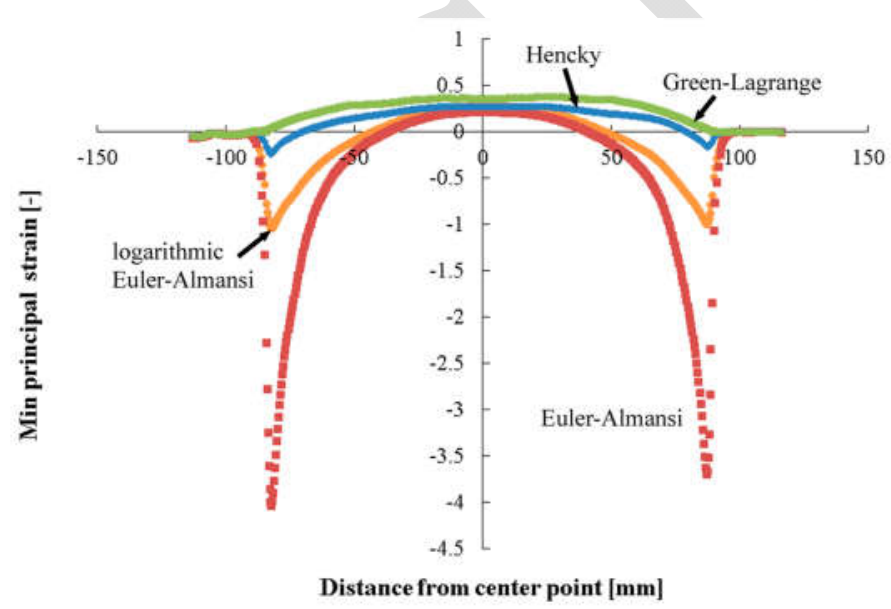

Figure 15. Minimum principal strain as calculated by VIC-3D 2009 as function of the distance to the centre 
Tables

\begin{tabular}{|l|c|c|}
\hline Correlation software & VIC-3D & MatchID3D \\
\hline Correlation algorithm & differences & differences \\
\hline Interpolation & Optimised 8-tap & Bicubic polynomial interpolation \\
\hline Stereo transformation & Not specified & Affine \\
\hline Correlation progress & Incremental & Spatial + update reference \\
\hline Subset weight / Noise handling & Gaussian & Gaussian \\
\hline Subset size [pixels] & $21 \times 21$ & 21 x 21 \\
\hline Step size [pixels] & 3 & 3 \\
\hline Filter size or strain window & 45 & 95 \\
\hline [pixels] & & \\
\hline Spatial resolution [pixels] & & \\
\hline
\end{tabular}

Table 1 Comparison of the correlation settings used in VIC-3D and MatchID3D 